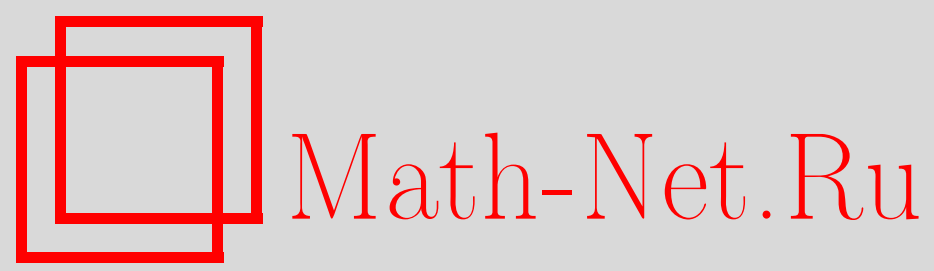

Л. А. Поморцев, В. И. Цурков, Перестройка порядков каскадно упорядоченного множества, Нечеткие системы и мягкие вычисления, 2020, том 15, выпуск 2, 96-115

DOI: https://doi.org/10.26456/fssc65

Использование Общероссийского математического портала Math-Net.Ru подразумевает, что вы прочитали и согласны с пользовательским соглашением

http://www.mathnet.ru/rus/agreement

Параметры загрузки:

IP: 18.234 .156 .22

26 апреля 2023 г., 14:26:09 


\section{УДК 51}

\section{ПЕРЕСТРОЙКА ПОРЯДКОВ КАСКАДНО УПОРЯДОЧЕННОГО MHOЖЕСТВА $^{1}$}

\section{Поморцев Л.А.*, Цурков В.И.**}

*Московский технический университет связи и информатики, г. Москва ** Федеральный исследовательский центр «Информатика и управление» Российской Академии Наук, г. Москва

Поступила в редакцию 02.02.2020, после переработки 31.08.2020.

В статье исследуются Частично и Каскадно Упорядоченные Множества (ЧУМ и КУМ) в аспекте развития алгоритмирования в Теории Реляционных Баз Данных (ТРБД). КУМ расширяет понятие ЧУМ за счёт включения в него цепочки вложенных друг в друга Частичных Порядков (ЧП). Примером КУМ служит Последовательность Вывода (ПВ) Функциональной Зависимости (Ф3) из заданной совокупности $\Phi 3$, в которой имеют место порядки следования и вывода одних $\Phi 3$ из других. Необходимость перестройки возникает в случаях повторов $\Phi 3$ в ПВ, которые преобразуются одной из Теорем настоящей работы в повторное их использование или, иными словами повторы какой-либо Ф3 заменяются исходящим из него гнездованием. Полученные результаты могут иметь самостоятельное значение в алгебре. $\mathrm{B}$ настоящей работе для доказательств применяются так называемые логические схемы.

Ключевые слова: силлогизм, анализ, синтез, таблица, отношение, атрибут, схема, ключ, функциональная зависимость, граф, логическая схема, кортеж, проекция, гнездование.

Нечеткие системы и мягкие вычисления. 2020. T. 15, № 2. С. 96-115. https://doi.org/10.26456/fssc65

\section{1. Введение}

\section{1. Целевые установки}

Мы продолжаем исследования, ведущие к алгебраизации Последовательности Вывода (ПВ) некоторой Функииональной Зависимости (ФЗ) из заданного множества других $\mathbf{\Phi 3}$, ради которых в [10] был задуман цикл статей, решающих следующие задачи ...

1. СИНТЕЗ-АНАЛИЗ последовательностей вывода $\mathbf{\Phi 3 ~ . . . . . . . . . . . . . ~ [ 1 1 ] ~}$

2. Перестройка порядков КУМ ..................... настоящая работа

3. Гигиена последовательностей вывода .................. продолжение

4. Алгебраизация последовательностей вывода ............... продолжение

${ }^{1}$ Работа выполнена при частичной финансовой поддержке РФФИ (грант № 19-01-00625). 
В задачах цикла, кроме прочего, устанавливаются необходимые методология исследований, терминология, а также первичные для данного цикла задач понятия и утверждения, которые в совокупности устанавливают логические правила обработки математических утверждений, придавая им форму ЛС. Статья развивает две темы: (1) Формализация доказательств утверждений;

(2) Перестройка порядков каскадно упорядоченного множества с приоритетами, соответствующими порядку перечисления. В порядке реализации темы (1) в доказательства утверждений статьи внедряются так называемые потоки предикатов и их обобщения ЛС, предназначеные для вычисления ПРЕДикАТОв (см. [11, гл.1,06зн.14 и §1.2 «Логические схемы»]).

В заключение необходимо отметь важную и неочевидную взаимосвязь двух тем данной статьи. Аристотелевский Kатегорический Cиллогизм (AKC) ${ }^{2)}$, на основе которых строится ЛС, удивительно похож на правило вывода В2 (накопление) [11, §2.5 «Аксиомы вывода»], реализующее по сути бинарную операцию. Все правила вывода и В2 в том числе составляют логический аппарат получения новых $\mathbf{\Phi 3}$ из заданных, а каждая ПВ является программой вывода, которая в свою очередь является примитивом ЛС. Сущности ЛС, ПВ и КУМ имеют одинаковую природу, несмотря на функциональные отличия. Это качество передаётся по наследству группе одноразовых действий, а именно: вывод в ЛС предикатов, вывод $\mathbf{\Phi 3}$ в ПВ, порядковое сравнение нижнего уровня в КУМ'е.

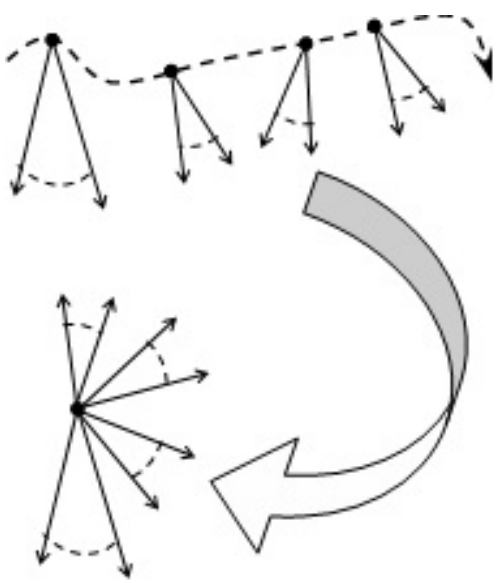
В задачах 3,4 цикла верхний уровень каскадного порядка (на рисунке слева это пунктирная линия, направленная в сторону возрастания порядка) реализует следование $\boldsymbol{\Phi} 3$ в ПВ. Звенья вывода $\mathbf{\Phi 3 ~ в ~ П В ~ ( н а ~ р и с . ~ и з о б р а ж е н ы ~ с т р е л - ~}$ ками) моделируют связи логического вывода в ЛС, которые в свою очередь управляют решением задачи настоящей статьи по преобразованию связей низлежащего порядка в КУМ'е, представляющих связи вывода $\mathbf{\Phi 3 ~ в ~ П В . ~ К р у г ~}$ замкнулся! Впрочем, это не круг, это спираль.

\section{2. Постановка задачи}

С левой стороны схематично показана постановка задачи 2 цикла и одновременно темы (2) данной статьи. В верхней части рисунка изображены конусы (см. далее Обзн.5) конечного Каскадно Упорядоченного Множества (КУМ), связанные пунктирной линией со стрелкой, изображающей некоторый его порядок. Нарисованные конусы порождаются следующим порядком и могут пересекаться, что осложняет задачу. Однако в результате перестройки связей порядка конусов они объединяются в его единый конус. Перестройка не влияет на высший порядок (обозначенный пунктиром) и затрагивает только рёбра, соединяющие опоры со следующей вершиной нижнего порядка. Объединительной порой в нижнем порядке становится опора первого конуса. Остальные опоры после перстройки уничтожаются. Исходные опоры называются повторами. Семантика этого слова будет восстановлена в задаче 3 . В статье глубина каскадности равна 2. Решение задачи 2 потребовало погружения КУМ в среду с глубиною 3. Несмотря на на это, каскадность можно сделать произвольной, благодаря универсальности полученного

\footnotetext{
2) Учение об $\mathbf{A K C}$ изложено Аристотелем в его Щервой аналитике» ( $\approx 350$ год до н. э.).
} 
решения.

В задачах 3,4 цикла роль КУМ'а играет ПВ с возможностью повторений некоторых $\boldsymbol{\Phi 3}$, что естественно. Устранение повторов позволяет упразднить принудительную адресацию $\mathbf{\Phi} 3$ в ПВ, но главное состоит в том, что решение задачи 4 цикла не допускает неоднозначности использования $\mathbf{\Phi} 3$. Volens nolens

3) с повторами приходится бороться и эта задача решается здесь заранее.

\section{3. О б о з н а ч е н и я: Иероглифы и Классы}

Под иероглифами понимаются обозначения, специальные символы и сокращения, необходимые для поддержки стиля изложения и структуры статьи.

ॠ мализованному и, в том числе, математическому;

Обзн. Обозначение ;

Опр. Определение ;

Кнст. Конструкция ;

Трм. Теорема.

для образования имён субъек-

тов цикла задач 1-4 и исполь-

Для придания смысловых акцентов на левых полях некоторых абзацев будут фигурировать метки, перечисленные ниже.

५ знак строгого предупреждения ${ }^{4)}$.

NB знак комментария, "мягкого" предупреждения;

Nota Bene, лат. $\sim$ хорошо заметить

Sic! факт не доказываемый в настоящей работе, непреложная истина. sic, лат. $\sim$ так.

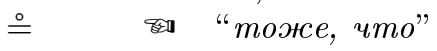

绻 С к о б к и , содержащие ссылку на субъекты ...

[ ] ॠ ... списка литературы

(см. ) ॠ ... настоящей работы

Под классом понимается множество однотипных понятий, объектов-субъектов (то есть сущностей). Классы обозначаются короткими полужирными словами формата маюскул ${ }^{5)}$. Обозначения классов используются в статье также для создания контекста в месте их расположения.

Примеры: КУМ, ТРБД, ПВ, ЛС и пр.

Прочие обозначения либо описаны в [10] и [11], либо формируются в статье по мере развития математического аппарата, решающего задачи цикла.

\section{2. Синтез-Анализ логических схем}

Большое внимание в цикле работ уделено автоматизации математического мышления. Начало этому направлению положено трудами Д.Гильберта и др., хотя достижения его теории, с которой можно познакомиться в монографиях [1] и [2] под общим названием «Основания математики», на сегодняшний день очень скромны по сравнению с целями, которые она перед собой ставила ${ }^{6)}$. Нас эта проблематика интересует с практической стороны. Для решения вопроса предлагается аппарат так называемых Логических Схем (ЛС), принципы функционирования которых описаны в [6]- [10] и особенно в [11]. В них же собрана

3) Volens nolens (лат.) - волей неволей

4) Знак \ в этом же смысле использует в своих трудах Н.Бурбаки (N.BOURBAKI)

5) Маюскул, маюскульное письмо (om лam. maiusculus - несколько больший) - алфавитное письмо, состоящее из прописных букв.

6) http://lib.mexmat.ru/books/1382 
начальная коллекция ЛС для дальнейшего их изучения, обобщения и теоретизации. В виду важности вопроса здесь мы продолжаем проникновение в алгоритмическую логику за счёт добавления к прежней коллекции новых ЛС, раскрывающих тему (2) настоящей статьи.

Статья [11], судя по названию, решала вопросы синтЕзА-АНАлизА применительно к ПВ. Однако СинТЕз, АнАлиз ${ }^{7)}$ являются философскими понятиями и на следующем этапе привлечения философии к математике в этой главе мы поднимаем планку исследований, расширяя их область за счёт Логических Схем (ЛС), являющихся объектами мышления. Это означает, что здесь мы обобщаем использование ЛС в работах [6]- [11] и предваряем их применение в текущей статье и в решениях оставшихся задач, предусмотренного выше цикла.

Внешне ЛС похожи на традиционные Блок-Схемъ (БС), используемые в информатике для алгоритмирования вычислительных процессов. Однако ЛС, в отличие от блок-схем, предназначены для вычисления предикатов [11, гл.1, 06зн.14] и могут и должы являться частью любого исскуственного интелекта. ЛС строятся на основе четырёх видов Разветвителей Логики (РЛ), один из которых представлен ниже в строке (1):

из $\mathscr{A}_{1}$ u $\mathscr{A}_{2}$ u ... и $\mathscr{A}_{k}$ cледует $\mathscr{A}$

где $\mathscr{A}_{1}, \mathscr{A}_{2}, \ldots, \mathscr{A}_{k}, \mathscr{A} \mid k \in \mathbb{Z}^{+}$- предикаты [11, 0бзн. 10,14], обобщающие количественно АKC - из $\mathscr{A}$ и $\mathscr{B}$ следует $\mathscr{C}$.

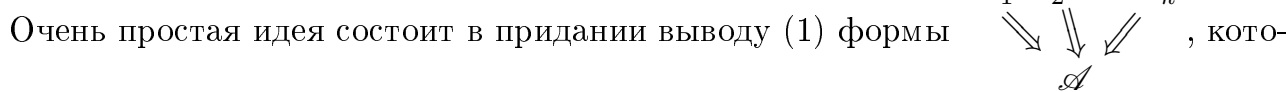
рую можно использовать как входящее гнездо в Графах Доказательств (ГД)|

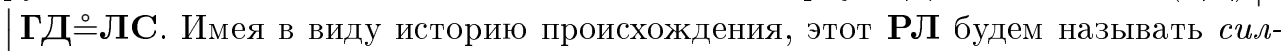
логизмом (без ярлыка категорический), сокращённо пСГ (прямой СиллоГизм).

$\mathscr{A}_{1} \mathscr{A}_{2} \ldots \mathscr{A}_{k}$

Та же идея $\mathbb{\nwarrow} \rrbracket$ относится к Одновременному Выводу Предикатов (OB) $\mathscr{A}_{1}, \mathscr{A}_{2}, \ldots, \mathscr{A}_{k}$ из предиката $\mathscr{A}$, реализующему композицию

$$
\text { из } \mathscr{A} \text { cледует } \mathscr{A}_{1} \text { u } \mathscr{A}_{2} \text { u } \ldots u \mathscr{A}_{k}
$$

OB изображает в ГД исходящее гнездо. С точки зрения мат.логики реализация OB не представляет собой каких-либо трудностей в отличие от силлогизма (1) в виде п्С , ибо $\left.\quad(2) \stackrel{\circ}{=}\left\{\mathscr{A} \Rightarrow \mathscr{A}_{1} u \mathscr{A} \Rightarrow \mathscr{A}_{2} u \ldots u \mathscr{A} \Rightarrow \mathscr{A}_{k}\right\}\right\} \stackrel{\circ}{=}$ ОВ .

Разветвители логики пСГ и ОВ строятся из предикатов, соединённых конгюнкиией, то есть союзом " $u$ " $\{\{$ тоже, что -И-, $\wedge,-$ AND- $\}\}$. Их достаточно для визуализации математиеской мысли, ибо союз “или” \{\{ тоже, что -Или-, $\vee$, $-\mathrm{OR}-\}\}$ можно реализовать за счёт предварительного разделения дидактических единниц на случаи и отображения каждого из них своим ГД. В качестве примера покажем, как союз -И- работает, расщепляя глаголы, роль которых в построении предикатов была выявлена в [11, гл.2,п.14]. Продемонстрируем это на бинарных предикатах вида $v \rho w$, где $\rho$ - глагол-отношение и $v, w$ - некоторые сущности. Аккуратность требует написать $v \in V, w \in W$ и $\rho \vec{\subset} V \times W$, где $V$ и $W$ множества сущностей, связываемых глаголом $\rho$. Пусть $\rho \stackrel{\circ}{=}-И-\beta$, тогда $v \alpha w \Rightarrow v \rho w \Leftarrow v \beta w$. Необходимо иметь в виду, что использование обозначения -И- диктуется грамматическим контекстом. Исходя из смыслового совпадения $-И-\stackrel{\circ}{=} \cap$ можно записать $\alpha-И-\beta \stackrel{\circ}{=} \alpha \cap \beta$. Выражение $\alpha \cap \beta$ будем использовать в математическом контексте.

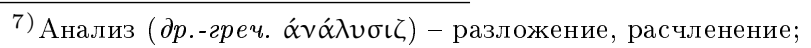

Синтез (дp.-греч. би́v $\theta \epsilon \sigma \iota)$ - соединение, складывание, связывание
} 
Разберём пример доказательства совпадения $S=T$ двух множеств $S$ и $T$. Оно порою строится из доказательств $S \subseteq T$ и $S \supseteq T$, помечаемых в статье маркерами $\subseteq$ и $\supseteq$ соответственно. Тоже относится к логической эвивалентности $\Leftrightarrow$. Доказательство утверждения $\mathscr{A} \Leftrightarrow \mathscr{B}$ разбивается на два этапа, открывающихся маркерами $\Rightarrow$ и $\Leftarrow$. Подытожим сказанное формулами

$$
=\stackrel{\circ}{=}-И-\supseteq \quad \text { и } \Leftrightarrow \stackrel{\circ}{=} \Rightarrow-И-\Leftarrow
$$

Проведём морфологический анализ двух выражений (3) для разъяснения принципа их чтения, требующего прежде всего выделения глагола в каждом предикате и затем тех сущностей, именуемых подлежащими, которыми он управляет: шаг 1: 을

это глагол ;

шаг 2: $=\subseteq-И-\supseteq \Leftrightarrow \Rightarrow-И-\Leftarrow$ $\notin 1$ это четыре подлежащих, являющихся шаг

3. $(A \subseteq B)-И-(A \supseteq B) \stackrel{\circ}{=}(\subseteq-И-\supseteq) B \stackrel{\circ}{=} A=B$

| проектный анализ глаго-

3: $(A \Rightarrow B)-И-(A \Leftarrow B) \stackrel{\circ}{=}(\Rightarrow-И-\Leftarrow) B \stackrel{\circ}{=} A \Leftrightarrow B$

лов в подлежащих шага

2 для сущностей $A$ и $B$.

Выше отмечалась возможность не использования дизъюнкций -Илидля построения разветвителей в ЛС, но всё же для отображения тонкостей математической мысли это сделать необходимо. Для исходящей дизъюнк-

ции за основу берётся инвертированный $\boldsymbol{C и л л о г и з м . ~ Ф о р м а л ь н о ~}$

$$
\prod_{\mathscr{A}}^{\mathscr{A}_{1} \mathscr{A}_{2} \ldots \mathscr{A}_{k}}
$$

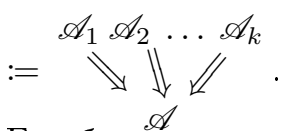

Ему будет соответствовать сокращение иСГ (инвертированный СиллоГизм). Инвертирование стрелок в выходном силлогизме позволит сохранить направление логического вывода в ГД (兰 $\mathbf{I C )}$.

Со Входящей Дизгюнкиией (ВД) всё проще. Достаточно согласиться, что

любое одиночное вхождение в $\searrow_{\mathscr{A}}^{\mathscr{A}_{1}} \mathscr{A}_{2} \ldots \mathscr{A}_{k}$ при условии корректности вывода $\mathscr{A}_{i} \Rightarrow \mathscr{A}$ и всего того, что входит в $\mathscr{A}_{i}$. Описанная здесь технология доказательств с одновременным использованием конъюнкций и дизъюнкций может быть проиллюстрирована Теоремой $3\langle 2\rangle$ в [1]].

Подведём итог:

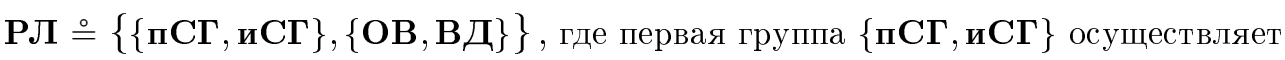
логические разделения предикатов на вХодЕ-Выходе соответственно, а вторая играет роль логических соединителей, как внутренних для ЛС, так и внешних между ЛС. В целом формирование ЛС по описанным правилам представляет собой Синтез сущностей, среди которых могут быть ранее сформированные ЛС.

Анализ какой-либо сущности на первом этапе заключается в выделении в ней некоторых (под)сущностей и стягивании их в точку (룰ину графа) вывода. В результате внутренние логические связи области стягивания станут невидимыми. Внешние связи при этом по-прежнему соединяют образованную точку с внешними (невыделенными) сущностями исходного ЛС. Возможность 
повторного применения к нему стягивания других вершин, включая вновь образованные, приведёт к графическому и логическому упрощению начального ЛС и будет результатом его анализа. Разумеется, вновь образованные точки вывода должны быть задокументированы в виде соостветствующих им ЛС на расстоянии шаговой доступности к результату анализа.

В собранном в процессе синтеза л.графе могут возникнуть циклы. Утверждения, находящиеся в них, логически эквивалентны. Совокупности, состоящие из них будем называть петлями. Они могут быть стянуты в точку логического вывода (兰 вершину л.графа). В результате целевых повторных стягиваний петель может/должен образоваться ациклический граф, представляющий логическую сущность исследуемого объекта.

Применение ЛС даёт авторам математических трудов возможность самопроверки, а читателям уверенность в корректности логического вывода результатов. Правильно построенная ЛС - это машина, её обмануть не возможно. Факт наличия ЛС в природе, но отсутствия в математике раскрывает причины невозможности создания полноценного искусственного интеллекта на основе традиционного алгоритмирования, использующего БС. Казалось бы логические основания в виде ЛС не имеют отношения к темам математических исследований задач 1-4, заявленным выше, однако они формируют её изложение и повышают доверие к полученным в них результатам. Необходимо понимать, что ЯЗЫК логических схем является неотъемлимой частью решения поставленных задач.

Синонимы: Логическая Схема (ЛС), л.схема, пространственный вывод,

Пространственный Алгоритм (ПА), Граф Доказательств (ГД) .

В математическом тексте ЛС изображаются диаграммами. Их внешний вид напоминает традиционные БС, однако это категорически разные средства искусственного интеллекта. Грубо говоря, БС служат целям управления данными, а ЛС, содержащие силлогизмы, моделируют мышление, несмотря на то, что пока силлогизм математически не формализован. Впрочем, начало этого можно усмотреть, как говорилось выше, в операции В2 (накопление) вывода $\mathbf{\Phi 3 . ~}$

Для автора-дизайнера представляется трудной технической задачей вписать ЛС в ограниченное графическое пространство и одновременно с этим вписать в него ранее сформированные понятия, ссылки на ЛС, процессы, а также математические объекты-субъекты (兰 сущности). Это делается на грани возможного и с этой целью последним придаётся вид обозначений, имеющих крайне экономные и выразительные формы, приближающие их к пиктограммам. Им в цикле работ уделяется особое и значительное внимание, поскольку на них держится понятийный аппарат математического текста.

\section{3. Бинарные отношения и частично упорядоченные множества}

\section{1. Бинарные Отношения в ТРБД}

Здесь, как и везде, под Бинарным Отношением (БО) имеется в виду классический объект алгебры, описанный, например, в [3, §2]. В цикле статей, упомянутых выше, вместе с этим употребляются понятие и термин “Плоско-Квадратное Отношение (ПКО)", введённые в [11, §2.80пр.16〈2〉] обобщающие БО и более приспособленные к нуждам Теории Реляционных Баз Данных (ТРБД). В алгебре не стоит вопрос о средствах взаимодействия БО между собой и с отношениями ( таблицами) общего вида, В ТРБД таким средством для каждого 
отношения является его схема (ㄹ шапка таблицы), состоящая из всех атрибутов отношения (兰 имён колонок таблицы). Схемы управляют алгебраической в сущности операцией $\bowtie$ соединения таблиц-отношений. Примитивным аналогом $\bowtie$ в алгебре является произведение пары БО. Если схему внести в БО, то получится ПКО. Обратно, если схема $X$ не бинарного отношения П удовлетворяет плоским требованиям $X=X^{1} \cup X^{2}$ и $X^{1} \cap X^{2}=\varnothing$, то отношение П можно понимать как бинарное, такое, что $\Pi \subseteq \operatorname{dom}\left(X^{1}\right) \times \operatorname{dom}\left(X^{2}\right)$. При этом требуется квадратность доменов $\operatorname{dom}\left(X^{1}\right)=\operatorname{dom}\left(X^{2}\right)$ для реализации произведения двух квази-БО, скажем П и Ш с совпадающими схемами $X:$ ПШ :=( $\left.X^{1} \Pi Z\right) \otimes\left(Z W X^{2}\right)[11, \S 2.8$, 0пр. $15\langle 2.2\rangle]$, где $Z:=X^{2}$ для П и $Z:=X^{1}$ для Ш. Благодаря [11, §2.8, Лемма 5 «Ассощиативность операщий $\bowtie$ и $\bigotimes \gg]$ операция • трактуется как умножение пары БО. Таким образом, в ПКО общего вида её плоско-квадратная схема $R$ имеет удвоенное количество $\mu\left(X^{1}\right)+\mu\left(X^{2}\right)=2 \mu\left(X^{1}\right)$ элементов и алгебра $\{\{\boldsymbol{\Pi K O}, R\}$, $, \otimes\}$ изоморфна алгебре $\{\mathbf{6 O}, \bullet\}$.

Выше речь шла о бинарной интерпретации отношений типа ПКО. Однако средствами ТРБД можно существенно расширить БО за счёт перехода к понятию "Квадратное Отношение" [11, §2.8 Опр. 15〈3〉], обобщающему ПКО. При этом результаты данной работы можно будет применить к отношениям $\rho \mid \rho:=$ П при условии соблюдения ими свойств РТА нижележащего Определения 1.

\section{2. Графы частично упорядоченных множеств}

Нижеследующие четыре объекта Опр.1,2 и Обзн.1,2 повторяют [11, 0пр.18,19] и $[11,06 з н$. 31,32] соответственно. Здесь они приводятся ещё раз для удобства чтения. $W$ - некоторое множество.

Определения 1. $\mathbf{P}$ T $\mathbf{C}$ А возможные свойства БО

\begin{tabular}{|c|c|c|c|c|c|}
\hline & Название свойства & Свойство & Пример & \multirow{5}{*}{$\begin{array}{l}5 \\
0 \\
5 \\
\vdots \\
\vdots \\
\end{array}$} & \multirow{5}{*}{$\rho, \sigma \vec{\subset} W^{\times 2}$} \\
\hline $\mathbf{P}$ & Рефлексивность & $\rho^{0} \subseteq \rho$ & $\rho:=\sigma^{0} \cup \vec{\subset} \sigma$ & & \\
\hline $\mathrm{T}$ & Транзитивность & $\rho^{2} \subseteq \rho$ & $\rho:=\sigma^{+}$ & & \\
\hline $\mathbf{C}$ & Симметричность & $\rho^{-1}=\rho$ & $\rho:=\sigma^{-1} \cup \sigma$ & & \\
\hline $\mathbf{A}$ & Антисимметричность & $\rho \cap \rho^{-1} \subseteq \rho^{0}$ & $\rho:=\left(\sigma \backslash \sigma^{-1}\right) \cup \vec{\subset} \sigma^{0}$ & & \\
\hline
\end{tabular}

Множество $\{\mathbf{P} \mathbf{T} \mathbf{C} \mathbf{A}\}$ является вертикальной схемой таблицы определения 1 , буквы которой используются для образования слов как в Обзн.1, символизирующих одновременное подчинение отношения свойствам наименованных ими.

Обозначение 1. Соединение свойств в слово

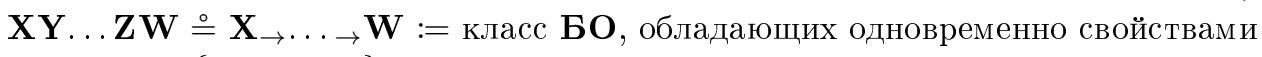
$\mathbf{X}, \ldots, \mathbf{W} \in\{\mathbf{P} \mathbf{T} \mathbf{C} \mathbf{A}\} \quad$. [11, 06зн.12.2]

Обозначение 2. Транзитно-конусное объединение БО $\mid \quad \rho, \sigma \vec{C} W^{\times 2}$ $\rho \uplus \sigma:=(\rho \cup \sigma)^{+} \equiv \bigcup_{k, l \in \mathbb{Z}+\times \infty} \prod_{i=0}^{\infty} \rho^{k_{i}} \sigma^{l_{i}} ; \Rightarrow \rho \uplus \sigma \in \mathbf{T} \quad$ \ поправка: $\rho^{0}:=\sigma^{0}:=(\rho \cup \sigma)^{0}$

Определения 2. Частично Упорядоченное Множество (ЧУМ)

$\langle\mathbf{1}\rangle \rho \mid\left\{\left\{\rho \subseteq W^{\times 2}\right.\right.$ и $\left.\left.\rho \in \mathbf{P T A}\right\}\right\}$ отношение Частичного Порядка (ЧП) ЧП вместо $\rho$ будет обозначаться через $\leqslant$ и $\geqslant$ или другими симметричными парами ассиметричных букв так, чтобы $\leqslant:=\rho$ и $v \leqslant w \stackrel{\circ}{=} \geqslant v \mid v_{\rightarrow} w \in W^{\times 2}$.

$\langle\mathbf{2}\rangle$ Пара множеств $\mathcal{W}:=\{W, \leqslant\}$ называется ЧУМ'ом

Обозначения 3. Алфавиты порядков 


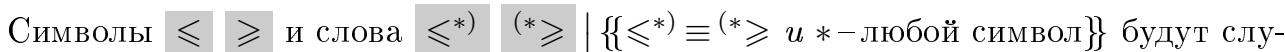
жить именами Частичных Порлджов (ЧП) на множестве $W$, где $W$ является
свободной переменной. В них знаки $\leqslant$ и $\geqslant$ также объявляются свободными буквенными переменными, которые могут быть замещены равнонаправленными знаками упорядочения, например $\leftrightharpoons$ или $\rightleftharpoons$ соответственно. Возможны также целочисленные вставки в имена ЧП:

通 $[11$, 0бзн. 5,10$]$

$$
\leqslant^{i)},{ }^{(i} \geqslant \mid\left\{\left\{i \in \mathbb{Z}^{+} u \leqslant{ }^{i)} \equiv{ }^{(i} \geqslant\right\}\right\}
$$

$\{\leqslant\}:=\left\{\leqslant^{i)} \mid i \in \mathbb{Z}^{+}\right\} \equiv\left\langle\leqslant^{0)}, \leqslant^{1)}, \ldots \leqslant \infty\right) ;\{\geqslant\}:=\left\{{ }^{(i} \geqslant \mid i \in \mathbb{Z}^{+}\right\} \equiv\left\langle\left\langle^{(0} \geqslant,{ }^{(1} \geqslant, \ldots(\infty \geqslant\rangle\right.\right.$ В соответствии с [11, Обзн. $27\langle 2\rangle$, Примечание] ЧУМ'ы задаются выражениями типа $\mathcal{W}:=\{W, \leqslant\} \quad, \quad \mathcal{W}:=\left\{W, \leqslant^{*)}\right\}$ или $\mathcal{W}:=\left\{W, \leqslant^{i)}\right\} \mid i \in \mathbb{Z}^{+}$

\ Символы $*$ и индексы $i \in \mathbb{Z}^{+}$играют роль меток в именах $\left.\leqslant *\right),\left(* \geqslant\right.$ и $\leqslant^{i)},{ }^{i} \geqslant$ плоских бинарных отношений, не являясь субъектами ЧУМ, в которьх они используются. Верхние скобочки необходимы для отличения меток от степеней отношения.

Обозначения 4. Экстремумы ЧУМ

$[11, \S 3.4$ Обзн. 35]

Все максимальные элементы $W$ в $\mathcal{W}$ $\max \mathcal{W} \doteq \max \{W, \leqslant\}:=\{w \mid w \in W$ и $w \geqslant x \mid x \in W\}$

Все минимальные элементы $W$ в $\mathcal{W}$ $\min \mathcal{W} \stackrel{\circ \min }{=} W, \leqslant\}:=\{w \mid w \in W$ и $w \leqslant x \mid x \in W\}$

С ин о н и мы:

$\{\{$ минимальный \{⿰элемент, параметр, операнд\}, точка входа, константа\}\}, $\{\{$ максимальный элемент, тупик, точка выхода, результат\}\}, $\{$ \{вХод, все минимальные элементы, констАнты \}, \{\{выход, все максимальные элементы\}\}.

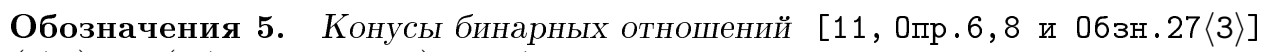
$(\vec{C} A) \rho:=((\vec{C} A \times A) \bowtie A \rho B) \vdash B \quad$ образы множеств $\vec{\subset} A, \vec{C} B$ отношения $\rho(\vec{C} B):=A \dashv(A \rho B \bowtie(B \times \vec{C} B)) \quad \rho \mid \rho \subseteq A \times B$ с возможностью замены $\vec{\subset}$ на $\in$. Если попроще и попонятнее, то $X \rho:=\{y \mid x \rho y, x \in X, y \in B\} \mid X \subseteq A$.

Аналогично

$$
\rho Y:=\{x \mid x \rho y, x \in A, y \in Y\} \mid Y \subseteq B .
$$

Термины 1 Правые и Левые конусы $(\vec{C} A) \rho-$ правый конус с опорой $\vec{C} A ; \rho(\vec{C} B)-$ левый конус с опорой $\vec{\subset} B$.

Определение 3. Линейно Упорядоченное Множество $\{W, \leqslant\}$ линейно упорлдочено, если оно является ЧУМ'ом и каждые два элемента из $W$ сравнимы. В этом случае отношение $\leqslant$ называется Линейным Порлдком (ЛП) в $W$. Его специальные обозначения $\lesseqgtr$ и

Sic! $\{W, \leqslant\}$ линейно упорядочено $\Longleftrightarrow W=w \leqslant U \leqslant w \mid w \in W$

Обозначения 6. Линейный порядок

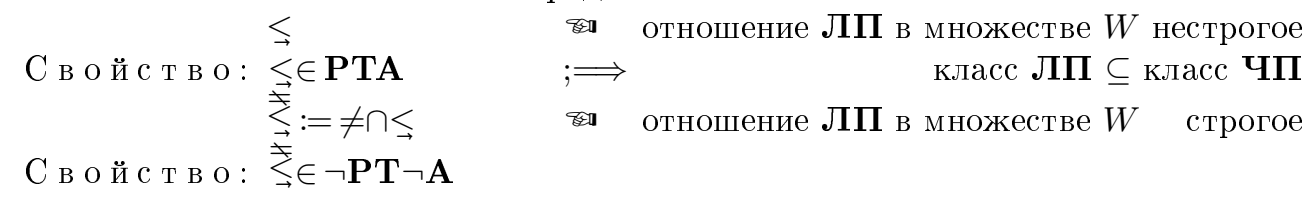

Обозначения 7. Графы [11, 06зн.34]

$\mathrm{B}$ настоящей работе рассматриваются только ориентированные (을 направленные) конечные графы (을 орарафы) из класса БО, но соглашения, принятые ниже относятся к произвольным графам:
$\langle\mathbf{1}\rangle \mathbb{G}$
множество вериин графа
$\langle\mathbf{2}\rangle \quad \gamma \mid \gamma \vec{C} \mathbb{G} \times \mathbb{G}$
множество рёбер графа
\... конечное
$; \Rightarrow \gamma \in \mathbf{6 O}$ на множестве $\mathbb{G}$ 
Далее отношения типа $\gamma \mid \gamma \vec{C} \mathbb{G}^{\times 2}$ таковы, что $\left\{\mathbb{G}, \gamma^{+}\right\}$являются частично или линейно упорядоченными множествами и $\gamma=\breve{\gamma}$. [11, Кнст. $1\langle 4,5\rangle]$

$\langle\mathbf{3}\rangle \quad \mathcal{G}:=\{\mathbb{G}, \gamma\}$ граф $[11,06$ зн.27 25$\rangle]$

$\langle\mathbf{4}\rangle \quad \mathcal{G}:=\ulcorner\gamma\urcorner$ опора графа (兰 все не изолированные вершины графа); $\mathcal{G} \mapsto\{\ulcorner\mathcal{G}\urcorner, \gamma\}$ минимизация графа

$\langle\mathbf{5}\rangle \mathbb{G} \backslash\ulcorner\mathcal{G}$ множество изолированных вершин графа

$\langle\mathbf{6}\rangle d(\mathfrak{g}, \mathcal{G}) \quad$ полустепень захода в вершину g графа $\mathcal{G}$ [4, §64, стр.283]

\ Ниже знак $\rightarrow$ используется в среде ЧУМ'ов и КУМ'ов в строгом соответствии с его определением [11, 06зн.12.2]. Это означает, что $\rightarrow$ символизирует разовый переход между элементами, вопреки связям порядка или напротив, используя их. Для произвольных множеств $Y, Z$ связь типа $\in Y_{\rightarrow} \in Z$ может быть кандидатом на включение её в порядок $\leqslant$ в виде ребра:

$\mathrm{NB}$

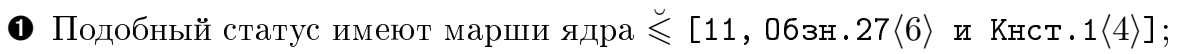

2 Если $y_{\rightarrow} z \in \leqslant$, то $y \leqslant z$, то есть согласованость символов $\rightarrow \leqslant$ характеризуется их разнонаправленностью. В общем случае начало $у$ и конец $z$ движения могут быть не сравнимы в $\{X, \leqslant\}$ и даже противоречить сравнимости.

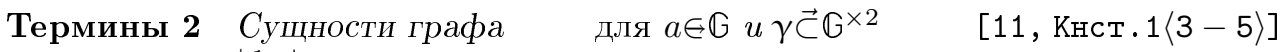
$\{a\} \cup a \gamma^{ \pm 1} \mid$ конус графа $\{\mathbb{G}, \gamma\}$ с корнем $a \quad(\stackrel{\circ}{=}$ опорой $a)$ $a \gamma^{ \pm 1}$ конус графа $\{\mathbb{G}, \gamma\}$ без корня а $\quad$ (если $\left.a^{\times 2} \notin \gamma\right)$

$\left.\begin{array}{l|l}a \gamma^{+} & \begin{array}{l}\text { конусы с } \\ \text { опорой } a\end{array}\end{array}\right\} a \gamma^{ \pm}=\gamma^{\mp} a$

$x \rightarrow a \gamma$ u $\gamma a_{\rightarrow} x \quad$ скелет конуса $a \gamma$ и $a \gamma^{-1}$ соответственно, $x-$ корень скелета $x_{\rightarrow} a \gamma^{+} u \gamma^{+} a_{\rightarrow} x \quad$ скелекон $\triangleq C К Е Л Е m$ КОНуса $a \gamma^{+}$и $a\left(\gamma^{+}\right)^{-1}$ соответственно

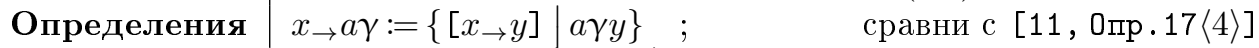
скелетов $\quad \gamma a_{\rightarrow} x:=\left\{\left[y_{\rightarrow} x\right] \mid y \gamma a\right\} \mid x$ любой субъект

Поясн ен и е : Под скелетом понимается новый по отношению к $\gamma$ граф образующийся соединением некоторого субъекта $x$ (взятого с потолка) со всеми элементами заданного конуса графа $\gamma$. Скелеты могу быть правыми и левыми в зависимости от того же качества применяемого конуса. (см. Термины 1)

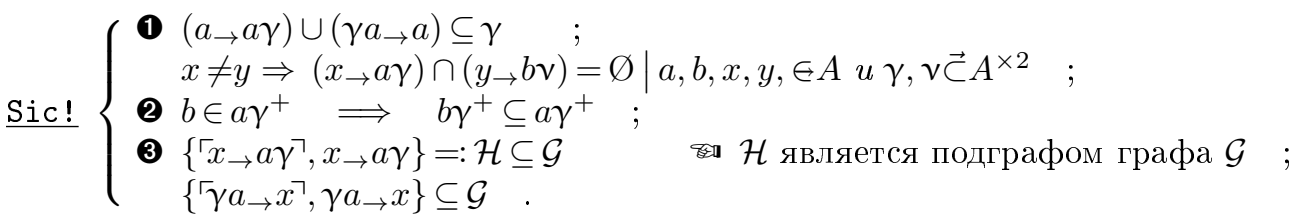

Термины 3 Типы скелеконов в ЧУМ'e $\{X, \leqslant\} \mid a, x \in X \quad$ (см. Термины 2)

\begin{tabular}{|c|c|c|}
\hline & Возрастающие скелеконы: & \\
\hline$a_{\rightarrow} x \leqslant$ и $\geqslant x_{\leftarrow} a$ & замкнутый скелекон & $a_{\rightarrow} x \leqslant \triangleq \geqslant \geqslant x_{\leftarrow} a$ \\
\hline$a_{\rightarrow} x<$ и & открытый скелекон & $a_{\rightarrow} x<\stackrel{\circ}{=}>x_{\leftarrow} a$ \\
\hline & Убывающие скелеконы: & \\
\hline$x \geqslant$ & замкнутый скелекон & $a_{\leftarrow} x \geqslant \stackrel{\circ}{=} \leqslant x_{\rightarrow} a$ \\
\hline$a_{\leftarrow} x>$ и $<x_{\rightarrow} a$ & открытый скелекон & $a_{\leftarrow} x>\stackrel{\circ}{=}<x_{\rightarrow} a$ \\
\hline
\end{tabular}

\ В обозначениях скелеконов частично упорядоченного множества используются только пары согласованных символов $\{\rightarrow, \leqslant\},\{\leftarrow, \geqslant\}$ и $\{\rightarrow,<\},\{\leftarrow,>\}$ - иное запрещено.

Обозначение 8. Разбиение множества (класса) $A \cup B:=A \cup B \mid A \cap B=\varnothing$

8) $\mapsto$ интервал рабочего времени, примитив порядка действий, шаг алгоритма [11, 0бзн.28]. См. также примечание 1. 
Лемма 1. Компенсация неправильности разового расширения ЧП (см.Опр.2) Если частично упорлдоченное множество $\{X, \leqslant\}$ конечно и $s, t \in X$, то $\leqslant \uplus\left\{s_{\rightarrow} t\right\} \in\{\mathbf{P T} \neg \mathbf{A}\} \Longrightarrow \leqslant \uplus\left\{s_{\leftarrow} t\right\} \in\{\mathbf{P T A}\} \quad$ (см. Опр.1, Обзн.1,2) Док-во. Операция $\uplus$ в любом случае приводит к справдливости условий $\mathbf{P T}$. Поэтому здесь мы акцентируем внимание на решении поставленной задачи в зависимости от состояния свойства $\mathbf{A}$. Кроме того

$$
\leqslant \uplus\left\{s_{\rightarrow} t\right\} \in\{\mathbf{P T} \neg \mathbf{A}\} \Rightarrow s \neq t \Leftarrow ; \leqslant \uplus\left\{s_{\rightarrow} s\right\} \in\{\mathbf{P T A}\} \Leftarrow ; \leqslant \uplus\left\{s_{\rightarrow} s\right\}=\leqslant
$$

Продолжим анализ компонент леммы, выделив в отдельное производство сравнимость элементов $s, t$ в частично упорядоченном множестве $\{X, \leqslant\}$. Пусть для определённости $s<t$. Пара $s \rightarrow t \mid:=s<t$ не удовлетворяет условию доказываемой ипликации, напротив пара $s_{\rightarrow} t \mid:=s>t$ ему удовлетворяет и вывод получается какой требуется, поскольку $s_{\leftarrow} t=s<t ; \Rightarrow \leqslant \uplus\left\{s_{\leftarrow} t\right\}=\leqslant ; \Rightarrow \leqslant \uplus\left\{s_{\leftarrow} t\right\} \in\{\mathbf{P T A}\}$.

Переходим к случаю $\left\{s_{\rightarrow} t, s_{\leftarrow} t\right\} \cap \leqslant=\varnothing$ несравнимости $s, t$ в $\{X, \leqslant\}$ :

Воспользуемся утверждением $\leqslant \uplus\left\{s_{\leftarrow} t\right\} \neg \in\{\mathbf{P T A}\} \Leftrightarrow \leqslant \uplus\left\{s_{\leftarrow} t\right\} \in\{\mathbf{P T} \neg \mathbf{A}\}\{\{\Leftarrow$ $\Leftarrow ;\{\mathbf{P T A}\} \bullet\{\mathbf{P T} \neg \mathbf{A}\}=\{\mathbf{P T}\}$ (см. Обзн.8) $\}\}$ и покажем, что условия

$$
(\bullet) \leqslant \uplus\left\{s_{\rightarrow} t\right\} \in\{\mathbf{P T} \neg \mathbf{A}\} \quad \text { и } \quad(\bullet) \leqslant \uplus\left\{s_{\leftarrow} t\right\} \in\{\mathbf{P T} \neg \mathbf{A}\}
$$

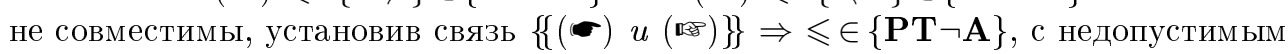
для частичного порядка $\leqslant$ выводом.

Обозначения $п у т и$ в бинарном отношении $\rho \vec{\subset} A^{\times 2}$

[11, 0пр.17]

из $a_{0}$ в $a_{n}$ через $\left.a_{i} \in A \mid i \in\right] 0, n$ [ и крайних точек пути:

$\left[a_{0} \rho_{a_{1}} \rho \ldots \rho_{a_{n}}\right]:=\left\langle\left[a_{0} \rho_{\left.a_{1}\right]},\left[a_{1} \rho_{a_{2}}\right], \ldots,\left[a_{n-1} \rho_{a_{n}}\right]\right\rangle=: \vec{a}\right.$ ]$\cdot \vec{a}:=a_{0} ; \vec{a} \cdot\left[:=a_{n}\right.$

крайние точки.

Истинность условия $(-)$ возникает при наличии в $\leqslant \uplus\left\{s_{\rightarrow} t\right\}$ колъцевых путей $\vec{p} \mid \vec{p} \cdot[=] \cdot \vec{p}$. Ими могут быть только пути, содержащие рёбра $\left[s_{\rightarrow} t\right]$, которые, кстати сказать, не могут в них соседствовать друг с другом по причине $s \neq t$. Прочие (некольцевые $\mid \stackrel{\circ}{=}$ разомкнутые) пути образуются исключительно отношением $\leqslant$. Это означает, что общий вид кольцевых путей даётся выражениями типа $\vec{p}$ :

$$
\begin{array}{ll}
p_{i j} \in X \mid i, j \in \mathbb{Z}^{+} & \Rightarrow \\
n_{i} \neq 0 \mid i \in[1, k[ & \Rightarrow \\
\vec{p} \cdot[=] \cdot \vec{p} & \Rightarrow
\end{array}\left\{\begin{array}{c}
\vec{p}:=\left[p_{01} \leqslant p_{02} \leqslant \ldots \leqslant p_{0 n_{0}} \leqslant s_{\rightarrow} t \leqslant\right. \\
\leqslant p_{11} \leqslant p_{12} \leqslant \ldots \leqslant p_{1 n_{1}} \leqslant s_{\rightarrow} t \leqslant \ldots \\
\left.\ldots \leqslant s_{\rightarrow} t \leqslant p_{k 1} \leqslant \ldots \leqslant p_{k n_{k}}\right]
\end{array}\right.
$$

Если ребро $s_{\rightarrow} t$ входит в $\vec{p}$ более одного раза, то вырезанный из него путь

$$
\vec{q}:=\left[s_{\rightarrow} t \leqslant p_{11} \leqslant p_{12} \leqslant \ldots \leqslant p_{1 n_{1}} \leqslant s\right]
$$

также является кольцом (兰 ииклом) в $\leqslant \uplus\{s \rightarrow t\}$, но более коротким и с единственным вхождением в $\vec{q}$ самодельного ребра $s_{\rightarrow} t$, которое к тому же является его началом. K этому будем стремиться и далее, но уже при условии единственного вхождения $s_{\rightarrow} t$ в $\vec{p}$.

Если $\vec{p}$ начинается с $s_{\rightarrow} t$ то есть $n_{0}=0$, то тот же $\vec{q}$ получается из $\vec{p}$ удалением хвостовой части сразу после $p_{1 n_{1}}$. Учитывая равенство $p_{1 n_{1}}=s$, получим вариант $\vec{q}$ без последнего ребра $p_{1 n_{1}} \leqslant s \mid=s \times s$, необходимости в котором нет в виду его ничтожности.

Остаётся рассмотреть случай $\vec{p}:=\left[p_{01} \leqslant \ldots \leqslant p_{0 n_{0}} \leqslant s_{\rightarrow} t \leqslant p_{11} \leqslant \ldots \leqslant p_{1 n_{1}}\right]$, когда $s_{\rightarrow} t$ входит в $\vec{p}$ один раз и при этом $n_{0} \neq 0$. Если $n_{1}=0$, то $p_{01}=t$ и $s_{\rightarrow} t$ можно поставить в начало, то есть перед $p_{01}$ и затем ликвидировать $p_{01}$. В результате получится цикл $\left[s_{\rightarrow} t \leqslant p_{02} \ldots \leqslant p_{0 n_{0}} \leqslant s\right]$, что ничем не хуже $(\downarrow)$, но есть опасность равенства $n_{0}=1$, которое ведёт к $\leqslant-$ сравнимости $s$ и $t$. Это было рассмотрено выше и является для нас пройденным этапом. В заключение рассмотрим условия $n_{0} \neq 0 \neq n_{1} . \Rightarrow p_{01}=p_{1 n_{1}} ; \Rightarrow\left[s_{\rightarrow} t \leqslant p_{11} \leqslant \ldots \leqslant p_{1 n_{1}} \leqslant p_{02} \ldots \leqslant p_{0 n_{0}} \leqslant s\right] \in$ $\in \leqslant \uplus\left\{s_{\rightarrow} t\right\}$. Это означает, что циклы отношения $\leqslant \uplus\left\{s_{\rightarrow} t\right\}$ с минимальным 
вхождением в них ребра $s_{\rightarrow} t$ имеют общий вид $(\diamond)$.

Подобный вывод на основании (桨) можно сделать для отношения $\leqslant \uplus\left\{s_{\leftarrow} t\right\}$. Выпишем для него минимальный цикл: $t_{\rightarrow} s \leqslant r_{0} \leqslant r_{1} \leqslant \ldots \leqslant r_{m} \leqslant t \quad$ и приведём его $\quad$ к виду $\vec{r}:=s \leqslant r_{0} \leqslant \ldots \leqslant r_{m} \leqslant t_{\rightarrow} s$. После соединения циклов $\vec{r}$ и $\vec{q}$ получим $\vec{r} \cdot \vec{q}=\left[s \leqslant r_{0} \leqslant \ldots \leqslant r_{m} \leqslant t_{\rightarrow} s\right] \cdot\left[s_{\rightarrow} t \leqslant p_{11} \leqslant \ldots \leqslant p_{1 n_{1}} \leqslant s\right]=\left[s \leqslant r_{0} \leqslant \ldots \leqslant r_{m} \leqslant\right.$ $\left.\leqslant t_{\rightarrow} s \cdot s \rightarrow t \leqslant p_{11} \leqslant \ldots \leqslant p_{1 n_{1}} \leqslant s\right]=\left[s \leqslant r_{0} \leqslant \ldots \leqslant r_{m} \leqslant t \leqslant p_{11} \leqslant \ldots \leqslant p_{1 n_{1}} \leqslant s\right] ; \Rightarrow$ $\Rightarrow \vec{r} \cdot \vec{q} \subseteq \leqslant ; \Rightarrow \leqslant \in\{\mathbf{P T} \neg \mathbf{A}\}\{\{\Leftarrow ; t \neq s\}$, что не есть возможно ; $\Rightarrow$ Лемма 1.

NB $\left\{\begin{array}{l}\checkmark \text { Конечности множества } X \text { и или частичного порядка } \leqslant \text { в Лемме } 1 \text { можно } \\ \text { избежать за счёт технического усовершенствования её доказательства. } \\ \checkmark \text { Требования } \leqslant \uplus\left\{s_{\rightarrow} t\right\} \in\{\mathbf{P T A}\} \text { и } \leqslant \uplus\left\{s_{\leftarrow} t\right\} \in\{\mathbf{P T A}\} \text { условно совмести- } \\ \quad \text { мы на фоне доказанной в лемме несовместимости } \leqslant \uplus\left\{s_{\rightarrow} t\right\} \in\{\mathbf{P T} \neg \mathbf{A}\} \\ \quad \text { и } \leqslant \uplus\left\{s_{\leftarrow} t\right\} \in\{\mathbf{P T} \neg \mathbf{A}\}\end{array}\right.$

Обозначения 9. Точки входа $\{\rtimes \Rightarrow, \Leftarrow \ltimes\}$ в ЛС и выхода $\{\Rightarrow \rtimes, \ltimes \Leftarrow\}$ из ЛС

Лемма 2. Краевые произведения скелекона ЧУМ'а $\{X, \leqslant\} \mid a, x \in X$

$$
\begin{aligned}
& \begin{array}{llll}
\mathbf{1} \leqslant \cdot\left(a_{\rightarrow} x \leqslant\right)=\leqslant a \times x \leqslant & ; & \mathbf{2} & \left(a_{\rightarrow} x \leqslant\right) \cdot \leqslant=a_{\rightarrow} x \leqslant \\
\mathbf{3} \leqslant \cdot\left(a_{\rightarrow} x<\right)=\leqslant a \times x< & ; & \mathbf{4} & \left(a_{\rightarrow} x<\right) \cdot \leqslant=a_{\rightarrow} x<
\end{array} \\
& \text { Док-во. (1) } s_{\rightarrow} t \in \leqslant \cdot\left(a_{\rightarrow} x \leqslant\right) \Leftarrow ; \Rightarrow s_{\rightarrow} t=s \leqslant a \cdot\left(a_{\rightarrow} t \mid x \leqslant t\right)=s_{\rightarrow} t \mid\{\{s \leqslant a u x \leqslant t\}\} \Leftarrow \text {; } \\
& \Leftarrow ; \Rightarrow s_{\rightarrow} t \in \leqslant a \times x \leqslant \\
& 2 \supseteq \leqslant \in\{\mathbf{P} \text { (рефлексивность }\} \Rightarrow a_{\rightarrow} x \leqslant \cdot \leqslant a_{\rightarrow} x \leqslant \\
& \subseteq \Leftarrow ; a_{\rightarrow} x \leqslant \ni a_{\rightarrow} t\left|x \leqslant t \Leftarrow ; s_{\rightarrow} t=\left(a_{\rightarrow} u\right) \cdot(u \leqslant t)\right| x \leqslant u \Leftarrow \\
& \text { (3) и } 4 \text { устанавливаются также } \\
& \Leftarrow s_{\rightarrow} t \in\left(a_{\rightarrow} x \leqslant\right) \cdot \leqslant \Leftarrow \ltimes
\end{aligned}
$$

Лемма 3. Усечение ЧП открытым скелеконом

(см. Термины 2)

Дано: $X$ - некоторое множество ; $a, x \in X ; \leqslant-$ частичный порядок на $X$

Требуется доказать: $\{X, \leqslant\} \in \mathbf{Y У М ~} \Longrightarrow\left\{X, \leqslant \backslash\left(a_{\rightarrow} a<\right)\right\} \in \mathbf{Y У М ~}$

Док-во. Отношение $\left.\leqslant^{a}\right):=\leqslant(a \rightarrow a<)$ наследует свойство $\mathbf{A}$ (антисимметричность) Определения 1 по причине $\leqslant^{a)} \subseteq \leqslant . \quad$ Кстати, $a \leqslant{ }^{a)} w \mid w \in X \Rightarrow a=w \Leftarrow$ $\Leftarrow \leqslant \in \mathbf{P}$ и $a_{\rightarrow} a \notin\left(a_{\rightarrow} a<\right)$. То есть для $\leqslant{ }^{a)}$ элемент $a$ является тупиком \{\{см. $[11, \S 3.4,06 з$. 35, , инонимы] . Ниже оно повторено в Обзн. 4$\}$. Рефлексивность $\mathbf{P}$ следует из пустоты $\leqslant 0 \cap(a \rightarrow a<)=\varnothing\{\{\Leftarrow ; a \notin a<\}\}$ (напомним $\leqslant 0=\ulcorner\leqslant$ [11, Кнст. $1\langle 3\rangle]$ ). Осталось убедиться в свойстве $\mathbf{T}$ (транзитивность) для $\leqslant^{a)}$ :

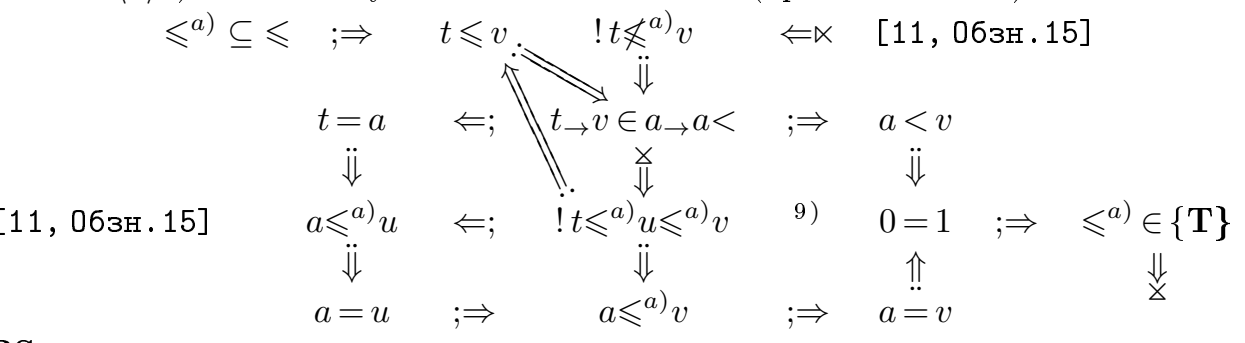

PS.

Здесъ имеет место первое применение ЛС в статве. Для сравнения представим доказательство Леммы 3, включая её ЛС, в традиционном виде:

Отметим очень простое свойство порядка $\leqslant^{a}$, вытекающее из его построения. Если $a \leqslant{ }^{a)} w$, то $a=w$, поскольку отношение $\leqslant$ рефлексивно и $a_{\rightarrow} a \notin\left(a_{\rightarrow} a<\right)$. То есть для $\leqslant$ ) элемент $a$ является тупиком. (см. ниже Синонимы в Обзн. 4)

9) Здесь и далее равенство $0=1$ обозначает состояние «противоречие» 
Пусть $t, u, v \in X$ такие, что $t \leqslant^{a)} u \leqslant{ }^{a)} v$. Из этого следует $t \leqslant u \leqslant v$ и, значит, $t \leqslant v$. Доказательство продолжим от противного, то есть предположим $t \Varangle^{a)} v$, но $t \leqslant v$. Следовательно $t_{\rightarrow} v \in a_{\rightarrow} a<$. Поэтому $t=a$. Отсюда $a \leqslant{ }^{a)} u$ и в силу тупиковости $a$ получим равенство $a=u$ и далее $a \leqslant^{a)} v$ и по аналогии $a=v$. Из ранее установленного включения $t_{\rightarrow} v \in a_{\rightarrow} a<$ выводим $a<v$, что противоречит равенству $a=v$. Источником противоречия является ранее сделанное предположение $t \Varangle^{v)}$, устраняя его получим $t \leqslant^{a)} v$, что означает транзитивность отношения $\leqslant^{a)}$.

\section{4. Каскадно Упорядоченное Множество}

$(\mathbf{K Y M})$

Определения 4. Каскады частичных порядков

$\langle\mathbf{1}\rangle$ Назовём множество $W$ базой $\mathbf{K У М , ~ а ~ с о в о к у п н о с т ь ~ Ч П ~}$

$\left\{\leqslant^{i)}\left|\leqslant^{i)} \subseteq W^{\times 2}\right| i \in\left[0, n[\}\right.\right.$ каскадом глубины $n \mid n \in \mathbb{Z}^{+}$на $W$, если

$$
v, w \in W \quad \Longrightarrow \quad\left\{\left\{v \leqslant^{i)} w \Rightarrow v \leqslant^{i-1)} w\right\}\right\} \quad \mid \quad i \in[1, n[
$$

Имея ввиду $[11,(2.5)]$, каскадность можно выразить включениями

$$
\leqslant^{0)} \supseteq \leqslant^{1)} \supseteq \cdots \supseteq \leqslant^{n-2)} \supseteq \leqslant^{n-1)}
$$

$\langle\mathbf{2}\rangle$ Обозначение КУМ'а глубины $n \mid n \in \mathbb{Z}^{+} \quad \mathcal{W}:=\{W,(4)\}$ $\mathcal{W} \triangleq\left\{W, \leqslant^{i-1)} \supseteq \leqslant^{i)} \mid i \in[1, n[\}\right.$ ॠаскадно Упорядоченное Множество $\mathcal{W}$ будем называть так же $n$-каскадным множсеством

$\langle\mathbf{3}\rangle$ Частично Упорядоченное Множество (ЧУМ) $\left\{W, \leqslant^{i)}\right\} \mid i \in[0, n$ [ будем называть $i$-тым слоем каскадно упорядоченного множества $W$.

Каскад это совокупность слоёв КУМ'а

\ ( МЫ ОГРАНИЧИМСЯ ДАЛЕЕ ГЛУБИНОЙ $n:=2$, то есть в основном ПодверГнем изучению 2-каскадные множества, но в качестве вспомогательной конструкции изредка будем использовать 3 -каскадное упорядочение.

(2) Напоминаем, мы имеем дело с конечными множествами, несмотря на универсальность Определения $4\langle 1\rangle$.

(3 Одновременное инвертирование порядков в $n$-каскадном множестве $\mathcal{W}$ приведёт к дуальной вариации $\mathcal{W}$, однако в настоящей работе они рассматриваться не будут.

$(4$ Нотация (4) для каскадных порядков будет основной

N B (1) Во всех слоях КУМ'а используется одно и тоже множество $\mathcal{W}$. Понятие «множество» не допускает повторов элементов в нём. Поэтому КУМ не может их иметь.

(2) Приведём здесь цитату из [3, Гл.1, §4,стр.17]: Всякая частичная упорлдоченность множества может быть продолжена до линейной упорлдоченности того же множества, то есть может быть включена в линейную упорлдоченность (в смысле включения бинарных отночений) [Шпильрайн, Fund. Math., 16 (1930), 386-389]. см. [13] Это означает, что любое ЧУМ можно рассматривать как 1-слой КУМ'а глубины 2, в котором 0-слой является ЛУМ'ом.

NB Теорема Цермело:

Всякое множество можно вполне упорлдочить .

$\Rightarrow$ Всякое конечное множество можно линейно упорядочить

Нижеследующая Лемма 4 выражает характеристическое свойство линейного порядка $\lesseqgtr$, которое могло бы быть его определением:

(см.Опр.3)

Лемма 4. Максимальность линейного порядка $\lesseqgtr \quad \Leftarrow$ Лемма 1 Собственное расширение полного порлдка приводит к нарушению условия $\mathbf{A}$

10) Замена термина "каскад" термином “цепь" лишит понятие полезной контекстности 


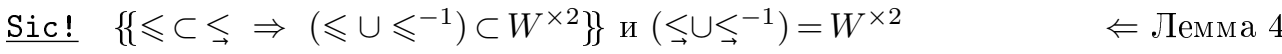

Ниже теоремой 1 на порядок $\leqslant$ произвольного частично упорядоченного множества $\{W, \leqslant\}$ накладывается линейный порядок $\leq$ :

Теорема 1. Конструктивное доказательство теоремы Шпильрайна для конечных ЧУМ

Любой частичный порядок $\leqslant \mid \leqslant \subseteq W^{\times 2}$ можно вложить в 2-каскад $\lesseqgtr \supseteq \leqslant$ Док-во. Построение строго возрастающей цепочки ЧП $\leqslant=: \leqslant^{0)} \subset \ldots \subset \leqslant^{i)} \subset \leqslant^{i+1)} \subset \ldots \subset \leqslant^{n)}=: \leq \mid i \in\left[0, n\left[\mid n \in \mathbb{Z}^{+}\right.\right.$

объемлющих заданный ЧП $\leqslant$ вплоть до линейного порядка $\lesseqgtr$ осуществим методом исключения (отсечения), основанным на Лемме 1, который в свою очередь, вложен в квази-графический алгоритм, исполненный ниже в традиционной для программирования манере:

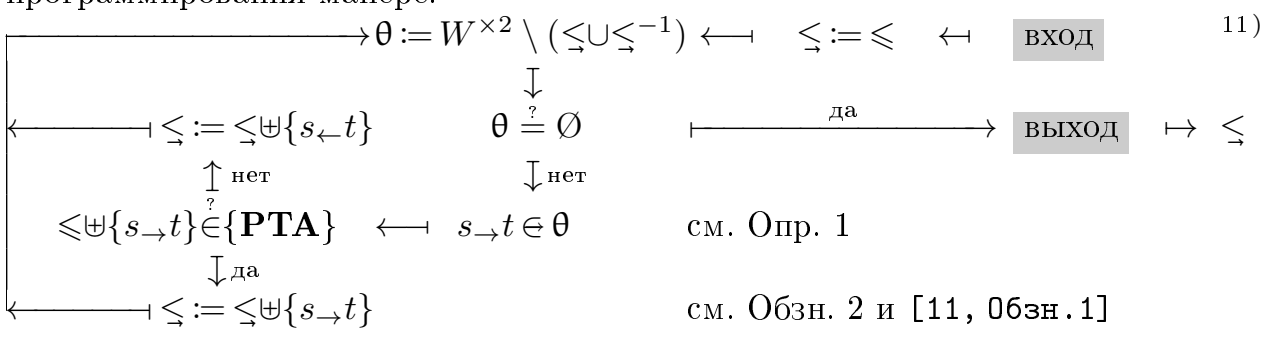

N В схеме отсутствуют "разделённые потоки сознания", основанные на силлогизмах $\left\{\left\{\mathscr{A}_{1} u \ldots u \mathscr{A}_{n}\right\}\right\} \Rightarrow \mathscr{B}$. Все блоки содержат информационно замкнутые действия. Из них исходят связи $\mapsto$, не помнящие родства, носящие управленческий характер ${ }^{12)}$. Необходимо понимать, ЛС категорически отличаются идейно и технологически от блок-схем, подобных представленной выше.

Кстати, отсутствие логических схем в программировании и адекватной элементной базы ЭВМ не позволяет современнной информатике широко выйти за пределы решения технических задач. Их примером может служить представленный в Теореме 1 алгоритм, формирующий новую сущность из существующих исключительно методами отбора и компановки, и только такие задачи составляют суть программирования. Разветвления в ЛС осуществляют прямые и обратные силлогизмы, что моделирует мышление. В настоящей статье они широко используются, но, к сожалению без понимания их сути, впрочем как везде. Разветвления в БС осуществляют булевские операторы, что значительно проще.

Синонимы: конструктивный, конечный

Теорема 2. Синхронная перестройка связей в порядках КУМ'a $\left\{X, \leqslant^{0)} \supseteq \leqslant \leqslant^{1)}\right\}$ $\langle\mathbf{0}\rangle \leqslant^{0)} \supseteq \leqslant^{1)} \longmapsto \quad \leq \supseteq \leqslant^{0)} \supseteq \leqslant^{1)} \quad$ (см. примечание 8)

$\langle\mathbf{1}\rangle$ Если $Y:=\left\{y_{0}, y_{1}, \ldots, y_{k}\right\} \vec{\subset} X \mid k \in[1, \mu(X)], \operatorname{mо~с~помощъю~} y_{0}:=\min \{Y, \lesseqgtr\}$ упорядоченности $\leqslant^{i)} \mid i \in\{0,1\}$ можно привести в состояния $\leqslant^{* i)} \mid\left\{\left\{\leqslant^{* i)} \in\{\right.\right.$ PТА $\} \quad$ (см.Опр.2) $\left.\}\right\}$, в которых совокупность скелеконов $\left\{y_{\rightarrow} y<^{i)} \mid y \in Y\right\}$ заменена на $y_{0 \rightarrow Y<}<^{i)}$, то есть:

通 (см. Обзн.2)

$$
\leqslant^{i)} \mapsto \leqslant^{* i)}:=\left(\leqslant^{i)} \uplus \bigcup_{j=1}^{k} y_{0 \rightarrow} y_{j}<^{i)}\right) \backslash \bigcup_{j=1}^{k} y_{j \rightarrow} y_{j}<^{i)} \mid i=0,1 ; k:=\mu(Y)-1
$$

11) На знак $\mapsto$ возложена функция передачи управления (см. примечание 8). Заметим, что в $\Rightarrow$ она присутствует изначально, но совместно с другими следствиями силлогизма, входящими в субъект вывода. $\mapsto$ имеет приоритет перед $\Rightarrow$

12 ) управленческий характер - “передать и забыть" 
$\langle\mathbf{2}\rangle \leqslant * 0) \supseteq \leqslant * 1)$

$\langle\mathbf{3}\rangle$ Множество $\left.\left\{X, \leqslant{ }^{* 0} \supseteq \leqslant * 1\right)\right\}$ является каскадно упорядоченным, как часть 3-каскадного множества $\{X, \leq \supseteq \leqslant * 0) \supseteq \leqslant * 1)\}$

Док-во. Упростим отношения $\leqslant^{\uplus i)}:=\leqslant^{i)} \uplus\left(\bigcup_{j=1}^{k} a_{\rightarrow} y_{j}<^{i)}\right) \mid\left\{\left\{i=0,1\right.\right.$ u $\left.\left.a:=y_{0}\right\}\right\}$ на основе представления операции $\uplus$ в Обозначении 2 . В соответствии с ним $\leqslant{ }^{\uplus i)}$ является объединением произведений различных степеней отношений из множества $\left\{\leqslant^{\uplus i)}\right\}:=\left\{\leqslant^{i)}, a_{\rightarrow} y_{j}<^{1)}, \ldots, a_{\rightarrow} y_{j}<^{k)}\right\}$. Ввиду рефлексивности $\left\{\left\{\Rightarrow \leqslant^{i) 0} \subseteq \leqslant^{i)}\right\}\right.$ и транзитивности $\{\{; \Rightarrow \leqslant i) 2=\leqslant i)\}$ неотрицательные степени отношения $\leqslant{ }^{i)}$ сводятся к 1 . Отношения $a_{\rightarrow} y_{j}<^{i)}$ и $a_{\rightarrow} y_{l}<^{i)}$ не соединимы при любых значениях $j, l \in[0, k]$, а с учётом утверждения 4 Леммы 2 они могут быть только последними буквами в словах алфавита $\left\{\leqslant^{\uplus i)}\right\}$ которые соответствуют компонентам развёрнутого отношения $\leqslant^{\uplus i)}$, причём в каждом таком слове может быть не более одного открытого скелекона вида приведённого выше.

$$
\begin{aligned}
& \Longrightarrow \quad \leqslant^{\uplus i)} \equiv \leqslant^{i)} \cup \bigcup_{j=1}^{k}\left(\leqslant^{i)} a \times y_{j}<^{i)}\right) \mid a:=y_{0} \\
& \Leftarrow \text {; (3 Лемма 2 и } a_{\rightarrow} y_{j}<^{i)} \subseteq \leqslant^{i)} a \times y_{j}<^{i)} \mid j \in[0, k] \Leftarrow \ltimes .
\end{aligned}
$$

Далее доказательство будем проводить в соответствии с планом, намеченном в формулировке Теоремы 2 :

$\langle\mathbf{1}\rangle$ Так же как в Лемме 3, которой по существу воспользуемся позже, операция $\backslash \cup_{j=1}^{k}$ не затрагивает диагоналей $\leqslant^{0)}$ и $\leqslant$ (1) $[11$, Кнст.1 $\langle 2\rangle]$, а операция $\uplus \cup_{j=1}^{k}$ не воспроизводит новых элементов в опорах $\left.\ulcorner\leqslant i)\right\urcorner=X \quad[11$, 06зн. 27 $\langle 5\rangle] . \Rightarrow \leqslant^{\uplus 0)}, \leqslant^{* 0)}, \leqslant^{* 1)} \in \mathbf{P}$ (рефлексивность). Свойство $\mathbf{T}($ транзитивность) для промежуточных отношений $\leqslant$ ४i) обеспечивается конусным расширением $+[11$, Кнст.1 $\langle 5\rangle]$, лежащим в основе транзитивного объединения $\uplus$ (см. Обзн.2).

尿 [11, 06зн.15]

$$
\text { Перейдём к } \mathbf{A} \text { (антисимметричность): }
$$

$\left.! t \leqslant{ }^{\uplus i)} u \leqslant \uplus i\right) t \mid\{\{i \in\{0,1\} ; \quad t, u \in X\}\}$. Интерес представляют бинарики 13) типа $t_{\rightarrow} u \mid t_{\rightarrow} u \notin \leqslant \leqslant^{i)} ; \Rightarrow t \rightarrow u \in \sigma^{i}:=$

$:=\bigcup_{j=1}^{k}\left(\leqslant^{i)} a \times y_{j}<^{i)}\right) ; \Rightarrow t \leqslant \leqslant^{i)} a$ и $y_{l}<^{i)} u ; \Rightarrow t \leq a$ и $y_{l} \stackrel{\ddagger}{\ddagger}\left\{\left\{\Leftarrow ; \leq \supseteq \leqslant^{0)} \supseteq \leqslant^{1)}\right\}\right\} ; \Rightarrow$ $\Rightarrow t \lesseqgtr a \lesseqgtr y_{l} \lesseqgtr u\{\{\Leftarrow ; a=\min \{Y, \lesseqgtr\}\}\} ; \Rightarrow t \lesseqgtr u$. На этом фоне исследуем две возможности (1) $u \leqslant{ }^{i)} t$ и (2) $u_{\rightarrow} t \in \sigma^{i}:$

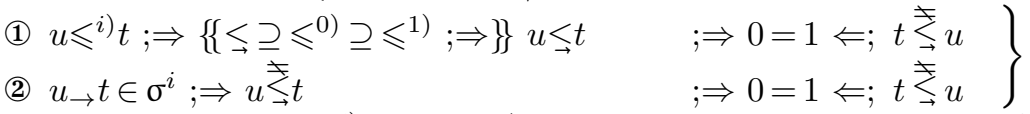

$$
\begin{aligned}
& \text { (2) } u_{\rightarrow} t \in \sigma^{i} ; \Rightarrow u \doteq t
\end{aligned}
$$

В итоге получаем $\leqslant{ }^{\uplus i)} \in\{$ PTA $\} \mid i \in\{0,1\}$, то есть отношения $\leqslant{ }^{\uplus 0)}$ и $\leqslant{ }^{\uplus 1)}$ являются частичными порядками. Переход от $\leqslant^{\uplus i)} \mathrm{K} \leqslant{ }^{* i)}$ с сохранением свойств PТА обеспечивается Леммой 3 и методом математической индукции по $k$.

$\langle\mathbf{2}\rangle \mathrm{У}_{\text {тверждение } \leqslant * 0) \supseteq \leqslant * 1)}$ докажем в три этапа, разделённых двумя горизонталными линиями. Результаты первых двух этапои отмечены серым фоном из них осуществляется выход из ЛС. Они будут использованы на заключительном этапе. Ниже приняты следующие обозначения: $a:=y_{0}, j \in[1, k]$.

$$
\begin{aligned}
& \left.\rtimes \Rightarrow \leqslant^{0)} \supseteq \leqslant^{1)} \Rightarrow \leqslant^{0)} a \supseteq \leqslant^{1)} a \quad \text { и } \quad y_{j}<^{0} \supseteq \supseteq y_{j}<^{1)} ; \Rightarrow \quad \leqslant^{0)} a \times y_{j}<0\right) \supseteq \leqslant{ }^{1)} a \times \\
& \times y_{j}<^{1)} \quad ; \Rightarrow \sigma^{0} \supseteq \sigma^{1} \quad ; \Rightarrow \quad \leqslant\left({ }^{\uplus 0} \supseteq \leqslant^{\uplus 1)} \quad \Leftarrow ; \leqslant^{\uplus i)}=\leqslant^{i)} \cup \sigma^{i} \mid i \in\{0,1\} \Leftarrow \ltimes\right. \\
& \Downarrow
\end{aligned}
$$

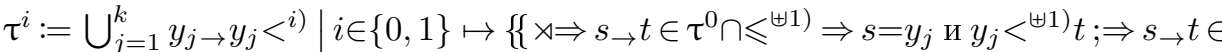

\footnotetext{
13) бинарики - элементы бинарного отношения

14) см. примечание 2
} 


$$
\begin{aligned}
& \in \tau^{1} ; \Rightarrow \tau^{0} \cap \leqslant^{\uplus 1)} \subseteq \tau^{1} ; \Rightarrow \tau^{0} \cap \leqslant^{\uplus 1)}=\tau^{1} \Leftarrow ; \tau^{0} \cap \leqslant{ }^{\uplus 1)} \supseteq \tau^{1} \Leftarrow ; \tau^{0} \supseteq \tau^{1} \Leftarrow \\
& \left.\left.\Leftarrow ; y_{j \rightarrow} y_{j}<^{0)} \supseteq y_{j \rightarrow} y_{j}<^{1)} \Leftarrow ; \leqslant^{0)} \supseteq \leqslant^{1)} \Leftarrow \ltimes\right\}\right\}
\end{aligned}
$$

Для доказательства заключительного этапа потребуется тривиальное тождество ( $)$, связывающее различные множества $A, B, X$ :

$$
\begin{aligned}
& \text { Sic! } \quad(X \backslash A) \cup(A \backslash B) \mid\{\{X \supseteq A \supseteq B\}\} \equiv X \backslash B \\
& \left.\left.\rtimes \Rightarrow \tau^{0} \cap \leqslant \uplus 1\right)=\tau^{1} ; \Rightarrow(\leqslant \uplus 1) \backslash \tau^{1}\right) \cap\left(\tau^{0} \backslash \tau^{1}\right) \underset{=\varnothing}{=\varnothing}
\end{aligned}
$$

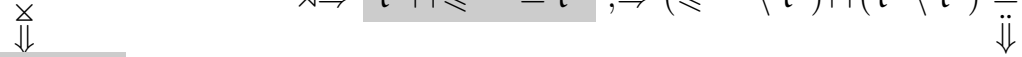

$$
\begin{aligned}
& \leqslant^{\uplus 0)} \supseteq \leqslant{ }^{\uplus 1)} ; \Rightarrow\left(\leqslant^{\uplus 0)} \backslash \tau^{0}\right) \cap\left(\tau^{0} \backslash \tau^{1}\right) \supseteq \leqslant^{\uplus 1)} \backslash \tau^{1} \quad ; \Longrightarrow \quad \leqslant^{\uplus 0)} \backslash \tau^{0} \supseteq \leqslant{ }^{\uplus 1)} \backslash \tau^{1}
\end{aligned}
$$

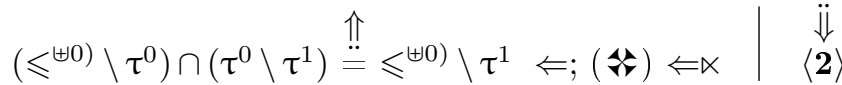

$\langle\mathbf{3}\rangle$ Включение $\lesseqgtr \supseteq \leqslant^{* 0)}$ является следствием определения $y_{0}:=\min \{Y, \lesseqgtr\}$ и внесения в состав $\leqslant$ () связей $\left.y_{0 \rightarrow} Y<0\right)$ для получения $\leqslant{ }^{* 0)}$.

$$
\begin{aligned}
& \Rightarrow\left\{\left\{z \in Y<^{0)} \mapsto y_{0} \leq y<<^{0)} z \mid y \in Y ; \Rightarrow y_{0} \leq y \lesseqgtr z\left\{\left\{\Leftarrow ; \leq \supseteq \leqslant^{0)}\right\}\right\} ; \Rightarrow\right.\right. \\
&\left.\left.\Rightarrow y_{0} \leq z ; \Rightarrow \leq \supseteq \leqslant^{* 0)} \Rightarrow \rtimes\right\}\right\} \Rightarrow \rtimes
\end{aligned}
$$

Термин 4 Повторы

Элементы из $Y \mid Y \vec{\subset} X$ будем называть повторами в КУМ'е $\left\{X, \leqslant^{0)} \supseteq \leqslant^{1)}\right\}$.

Казалось бы выбор $Y \vec{C} X$ зависит от воли оператора, но всё же термин “Повторы" необходим для кластеризации тех $\mathbf{\Phi 3 , ~ к о т о р ы е , ~ с о в п а д а я ~ м е ж д у ~ с о - ~}$ бой, фигурируют в разных местах ПВ. К сожалению непрограммные (математические) критерии образования подобных кластеров в абстрактных КУМ'ах отсутствуют.

Ниже речь идёт о перестройке порядка $\leqslant$ 1) 2 -каскадного множества без измений его основы $\leqslant^{0)}$ в случае её линейности $\leqslant^{0)}=\leq$ :

Следствие т-2 Консолидация повторов 2-каскадного множества с линейной основой $\leqslant$ )

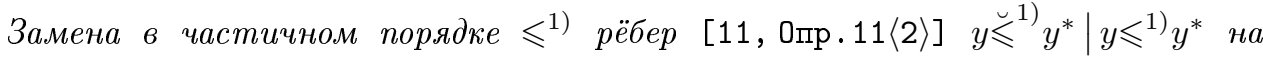
$\min \{Y, \leq\} \rightarrow y^{*}$ перестраивает порядок $\left.\leqslant 1\right)$, преобразуя его в частичный поря$\partial o \kappa \leqslant * 1) \mid \leq \supseteq \leqslant * 1)$

Определение 5. Точка плотности

Элемент $\theta$ КУМ'а $\mathcal{W}$ назовём $i$-nлотным $\mid i \in\left[0, n\left[\right.\right.$, если в левом конусе $\leqslant^{i-1)} \theta$ нет тупиков слоя $\leqslant$ кроме $\left.\theta \stackrel{\max }{=} \leqslant \leqslant^{i-1)} \theta, \leqslant^{i)}\right\}=\{\theta\}$

П о я с н е н и е : Выражение $\max \left\{\leqslant^{i-1)} \theta, \leqslant^{i)}\right\}$, фигурирующее в Опр.5, соответствует синтаксису Обзн.5: $\leqslant^{i-1)} \theta-$ левый конус элемента $\theta \mid \theta \in W$. Это значит, что $\leqslant{ }^{i-1)} \theta \subseteq W$, но на $\leqslant^{i-1)} \theta$, кроме $\leqslant^{i-1)}$, работает также отношение $\leqslant^{i)}$ поскольку $\mathcal{W} \stackrel{\circ}{=}\left\{W, \leqslant^{0} \supseteq \leqslant^{1)} \supseteq \ldots \supseteq \leqslant^{n-2)} \supseteq \leqslant^{n-1)}\right\}-$ каскадно упорядоченное множество. В результате получаем ЧУМ $\left\{\leqslant^{i-1)} \theta, \leqslant^{i)}\right\}$, в котором есть законное подмножество $\max \left\{\leqslant{ }^{i-1)} \theta,{ }^{i)}\right\}$.

Sic! Двухкаскадное множество располагает всего одним вариантом - 1 плотность, который упрощённо будем называть плотностью

Синонимы: точка плотности, плотная(ый) \{точка, элемент и пр.\}

Лемма 5. Критерий плотности $\theta \mid \theta \in W$ в двухкаскадном множестве $\mathcal{W}$ $\theta$ плотен в $\mathcal{W}:=\left\{W, \leqslant^{0)} \supseteq \leqslant^{1)}\right\} \Longleftrightarrow \leqslant^{0)} \theta=\leqslant^{1)} \theta \quad$ (см. "конус" в Термины 2) Док-во. $\leqslant{ }^{0)} \supseteq \leqslant{ }^{1)} \Rightarrow \leqslant{ }^{0)} \theta \supseteq \leqslant{ }^{1)} \theta$. Поэтому достаточно установить 


$$
\begin{aligned}
& \theta-\text { плотен } \Longleftrightarrow \quad \leqslant^{0)} \theta \subseteq \leqslant^{1)} \theta \\
& x \in \leqslant^{0)} \theta \Rightarrow x \leqslant^{1)} \theta ; \Rightarrow x \in \leqslant^{1)} \theta\left\{\{\Leftarrow ; \theta \text { плотен в } \mathcal{W}\} ; \Rightarrow \leqslant^{0)} \theta \subseteq \leqslant^{1)} \theta\right. \\
& \text { тавтология }
\end{aligned}
$$

Лемма 6. Уплотнение элемента в двухкаскадном множестве

Двухкаскадное множество $\mathcal{W} \mid \leqslant^{0)} \supseteq \leqslant^{1)}$ за счёт измнения порядка элементов в нижнем слое $\left\{\mathcal{W}, \leqslant^{0)}\right\}$ и сохранения порядка в верхнем слое $\left\{\mathcal{W}, \leqslant^{1)}\right\}$ можно привести в состолние, в котором произвольно выбранный элемент $\omega \in \mathcal{W}$ станет плотным.

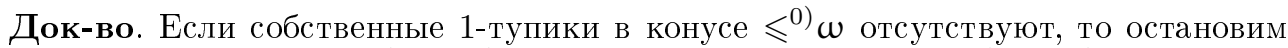
процесс, иначе $\max \left\{\leqslant^{0)} \omega, \leqslant^{1)}\right\} \backslash\{\omega\} \neq \varnothing ; \Rightarrow ! \omega_{0} \in \max \left\{\leqslant^{0)} \omega, \leqslant^{1)}\right\} \backslash\{\omega\} \quad[11$, 0бзн. 15 и 0пр. $17\langle 1-3\rangle]$. Переведём сделанную запись на традиционный язык: Если в конусе $\leqslant 0) \omega$ есть собственные 1-тупики, то выберем один из них, скажем $\omega_{0}$. И далее переместим его за пределы конуса $\leqslant^{0)} \omega \backslash \omega_{0}$, сделав его $\leqslant^{0)}$ максимальным. Иными словами осуществим переход

$$
\left[\leqslant^{0)} \omega\right] \mapsto\left[\left[\leqslant^{0)} \omega \backslash \omega_{0}\right] \leqslant{ }^{0)} \omega_{0}\right] .
$$

Этот процесс можем продолжать, пока не исчерпаем все 1-тупики вместе с источниками их возникновения. То есть в общем случае образуется путь вида

$$
\left.\mathcal{K}:=\left[\left[\leqslant^{0)} \omega\right] \backslash\left\{\omega_{0}, \ldots \omega_{n}\right\} \leqslant^{0)} \omega_{0} \leqslant{ }^{0}\right) \ldots \leqslant \leqslant^{0)} \omega_{n}\right] \mid 0 \leqslant n \leqslant \mu\left(\left[\leqslant^{0)} \omega\right]\right)
$$

Целочисленный параметр $n$ определяется общей глубиной проникновения 1тупиков в их конусный скелет $\left[\leqslant^{0)} \omega\right]$ в направлении обратном к $\leqslant$. . Совпадение составов $\ulcorner\mathcal{K}\urcorner=\left\ulcorner\left[\leqslant^{0)} \omega\right]\right\urcorner$ и выход связей $\omega_{i} \leqslant^{1)} \mid i \in[0, n]$, которых мы не трогали, для обоих конусов за их пределы в направлении возрастания $\leqslant 1$ даёт основание заменить в $\mathcal{W}$ фрагмент $\left[\leqslant^{0)} \omega\right]$ строго соответствующим ему фрагментом $\mathcal{K}$.

В результате получим двухкаскадное множество $\mathcal{W}^{\leqslant^{1)} \omega}$, в котором элемент $\omega$ будет плотным.

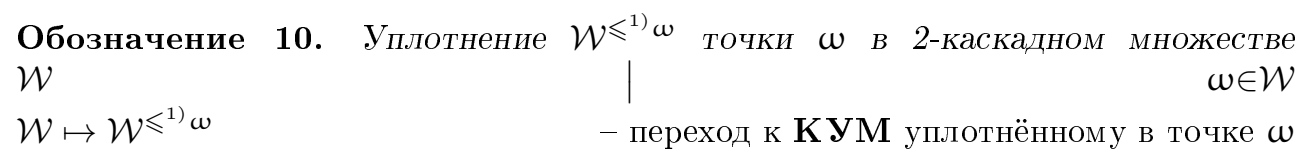

\section{5. Заключение}

В представленной статье проделана подготовительная работа по нормализации ПВ, которые, как было сказано, являются разновидностью вычислительных программ, обрабатывающих заданную совокупность $\mathbf{\Phi 3}$ и получающих на выходе

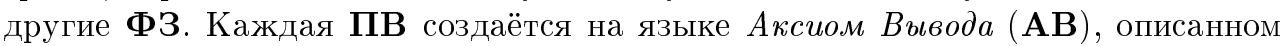
в [10, гл.2] и [11, раздел 2.5]. Качество ПВ, как и всякой программы, зависит, в том числе, от вне математических факторов и может меняться в ту или другую сторону в процессе её разработки и последующей отладки. Каждый программист знает, что первый вариант созданной программы неработоспособен и необходима дальнейшая работа над ней, которая называется отладкой (을 debug). В её процессе корректируется постановка задачи, устраняются синтаксические ошибки, а также вносятся изменения в связи управления программным кодом без нарушения целевого назначеия программы. Последнее осуществляется частично в рамках темы «Перестройка порядков каскадно упорядоченного множества» настоящей работы, но, судя по уровню абстракции решения, она может быть применима не только для ПВ. Следующим этапом наших исследований станет в задаче 3 цикла формализация отладки ПВ, использующая полученные здесь результаты. Прежде всего речь пойдёт об очистке ПВ от программного 
мусора, что в известном смысле минимизирует программный код. Затем минимизация будет реализована за счёт устранения повторов использования $\mathbf{\Phi} 3$, как это предусматривается Следстием Т-2, что ведёт к образованию (см. [4]) Исходящего Гнездования (ИС) Ф3, если ПВ рассматривать как граф вывода. Однако с точки зрения минимизации устранение повторов является полумерой. К счастью констуктивные особенности $\mathbf{\Phi} 3$ и ПВ позволяют существенно большее, а именно возможно упразднеие ИС в самом выводе. Это в конечном счёте приведёт к алгебраизации ПВ, но сильно потом - в задаче 4 цикла, упомянутого во Введении. Задача 3 «Гигиена последовательностей вывода» формализует отладку ПВ, но поскольку ПВ, являясь программой, всё же ей не яляется в общеупотребительном смысле, так как не содержит команд передачи управления, то термин Отладка заменён словом Гигиена. Это больше соответствует нашему намерению наведения чистоты и порядка в доме, именуемом ПВ, что предписывает использование стиля ALT [10, 0бзн.12 «Стили вывода»] при создании ПВ, в котором исходные и полученные $\boldsymbol{\Phi} 3$ сторого чередуются с командами вывода, укладываясь в линейную последовательность.Таким образом задача 3 Введения позволяет преобразовать ПВ в Функиионально Эквивалентную (ФЭ) (см. [10] и [11]) ей чередующуюся последовательность вывода, от которой рукой подать до алгебраического выражения, делащего ту же работу, что и исходная ПВ.

\section{Список литературы}

[1] Гильберт Д., Бернайс П. Основания математики. Логические исчисления и формализация арифметики. М.: Наука, 1979.

[2] Гильберт Д., Бернайс П. Основания математики. Теория доказательств. М.: Наука, 1982.

[3] Курош А.Г. Лекции по общей алгебре. М.: Физматлит, 1962.

[4] Емеличев В.А., Мельников О.И., Сарванов В.И., Тышкевич Р.И. Лекции по теории графов. М.: Наука, 1990.

[5] Мейер Д. Теория реляционных баз данных. М.: Мир, 1987.

[6] Поморцев Л.А. Вычисление моментов порядковых статистик последовательных сумм симметрически зависимых случайных величин // Труды Московского математического общества. 1983. Т. 46. С. 201-242.

[7] Поморцев Л.А. Комбинаторно-симметрический анализ многомерных случайных блужданий // Дискретная математика. 1991. Т. 3, № 1. С. 21-41.

[8] Поморцев Л.А. Алгебраическая интерпретация полноты аксиом вывода // Фундаментальная и прикладная математика. 2002. Т. 8, № 1. С. 195-219.

[9] Поморцев Л.А. Алгебры функциональных зависимостей в теории реляционнных баз данных // Труды Института Системного анализа РАН. Динамика неоднородных систем. 2007. Т. 29, № 1. С. 169-183.

[10] Поморцев Л.А., Цурков В.И. Алгебраизация вывода функциональных зависимостей реляционных баз данных // Известия РАН. Теория и системы управления. 2019. № 2. С. 58-74. https://doi.org/10.1134/S000233881902015X 
[11] Поморцев Л.А., Цурков В.И. СИНТЕЗ-АНАЛИЗ последовательностей вывода функциональных зависимостей таблиц // Известия РАН. Теория и системы управления. 2020. Т. 6. С. 152-176.

[12] Яблонский С.В. Введение в дискретную математику. М.: Наука, 1986.

[13] Szpilrajn E. Sur l'extension de l'ordre partiel // Fundamenta Mathematicae. 1930. Vol. 16. Pp. 386-389. https://doi.org/10.4064/fm-16-1-386-389

\section{Образец цитирования}

Поморцев Л.А., Цурков В.И. Перестройка порядков каскадно упорядоченного множества // Нечеткие системы и мягкие вычисления. 2020. Т. 15, № 2. C. 96-115. https://doi.org/10.26456/fssc65

\section{Сведения об авторах}

\section{1. Поморцев Леонид Александрович}

доцент кафедры информатики Московского технического университета связи и информатики.

Россия, 123423, г. Москва, Северо-Западный Административный Округ, ул. Народного Ополчения, д. 32, МТУСИ. E-mail: brickfactory.org@таil.ru

\section{2. Цурков Владимир Иванович}

заведующий отделом ФИЦ ИУ РАН.

Россия, г. Москва, ул. Вавилова, д.44, корп.2.E-таil: tsur@сcаs.ru 


\title{
ORDER ALTERATION IN A CASCADE-ORDERED SET
}

\author{
Pomortsev Leonid Alexandrovitch \\ Associate Professor of the Department of Informatics, Moscow Technical University \\ of Communications and Informatics \\ Russia, 123423, Moscow, North-Western Administrative District, 32 Narodny \\ Opolcheniya str., MTUSI. \\ E-mail: brickfactory.org@mail.ru \\ Tsurkov Vladimir Ivanovich \\ Head of the Department, Federal Research Center "Computer Science and \\ Control"of Russian Academy of Sciences \\ Russia, Moscow, 44 Vavilova str., build. 2. \\ E-mail:tsur@ccas.ru
}

Received 02.02.2020, revised 31.08.2020.

The article examines the Partially and Cascade of Ordered Sets (POS and COS) in aspect of the development of algoritms in the Theory of Relational Databases (TRDB). COS expands the notion of POS by including himself a chain of Partial Orders (PO) wich nested into each other. An example of COS is the Sequence of the Derivation (SD) of the Functional Dependence (FD) from a given set of FDs which have two orders of following and of derivation of some FD from others. The need for restructuring arises in cases of repetitions of the $\mathrm{FD}$ in $\mathrm{SD}$, which are transformed into their reuse or, in other words, replicas of any FD are replaced by the outgoing from him nesting. The received results can have an independent value in algebra. In the present paper, so-called logic schemes are used for proofs.

Keywords: syllogism, analysis, synthesis, database table, relation, attribute, scheme, key of table, functional dependence, graph, logical scheme, cortege, projection, nesting.

\section{Citation}

Pomortsev L.A., Tsurkov V.I., "Order alteration in a cascade-ordered set", Nechetkie Sistemy i Myagkie Vychisleniya [Fuzzy Systems and Soft Computing], 15:2 (2020), 96-115(in Russian). https://doi.org/10.26456/fssc65

\section{References}

[1] Gilbert D., Bernajs P., Osnovaniya matematiki. Logicheskie ischisleniya i formalizatsiya arifmetiki [Foundations of mathematics. Logical calculus and formalization of arithmetic], Nauka Publ., Moscow, 1979 (in Russian).

[2] Gilbert D., Bernajs P., Osnovaniya matematiki. Teoriya dokazatelstv [Foundations of mathematics. Proof theory], Nauka Publ., Moscow, 1982 (in Russian).

[3] Kurosh A.G., Lektsii po obshchej algebre [Lectures on general algebra], Fizmatlit Publ., Moscow, 1962 (in Russian). 
[4] Emelichev V.A., Melnikov O.I., Sarvanov V.I., Tyshkevich R.I., Emelichev [Lectures on Graph Theory], Nauka Publ., Moscow, 1990 (in Russian).

[5] Mejer D., Teoriya relyatsionnykh baz dannykh [The theory of relational databases], Mir Publ., Moscow, 1987 (in Russian).

[6] Pomortsev L.A., "Calculation of moments of ordinal statistics of consecutive sums of symmetrically dependent random variables", Trudy Moskovskogo matematicheskogo obshchestva [Proceedings of the Moscow Mathematical Society], 46 (1983), 201-242 (in Russian).

[7] Pomortsev L.A., "Combinatorial-symmetric analysis of multidimensional random walks", Diskretnaya matematika [Discrete mathematics], 3:1 (1991), 21-41 (in Russian).

[8] Pomortsev L.A., "Algebraic interpretation of the completeness of the axioms of inference", Fundamentalnaya $i$ prikladnaya matematika [Fundamental and Applied Mathematics], 8:1 (2002), 195-219 (in Russian).

[9] Pomortsev L.A., "Functional dependency algebras in relational database theory", Trudy Instituta Sistemnogo analiza RAN. Dinamika neodnorodnykh sistem [Proceedings of the Institute of System Analysis of the Russian Academy of Sciences. Dynamics of inhomogeneous systems], 29:1 (2007), 169-183 (in Russian).

[10] Pomortsev L.A., Tsurkov V.I., "Algebraization of inferring functional dependences in relational databases", Journal of Computer and Systems Sciences International, 58:2 (2019), 212-228, https://doi.org/10.1134/S000233881902015X.

[11] Pomortsev L.A., Tsurkov V.I., "SYNTHESIS AND ANALYSIS OF sequences of output of functional dependencies of tables", Journal of Computer and Systems Sciences International, 6 (2020), 152-176.

[12] Yablonskij S.V., Introduction to Discrete Mathematics, Nauka Publ., Moscow, 1986 (in Russian).

[13] Szpilrajn E., "Sur l'extension de l'ordre partiel", Fundamenta Mathematicae, 16 (1930), 386-389, https://doi.org/10.4064/fm-16-1-386-389. 